DRAFT VERSION JULY 23, 2018

Preprint typeset using LTEX style emulateapj v. 5/2/11

\title{
REAPPROACHING THE SPIN ESTIMATE OF GX 339-4
}

\author{
R. M. Ludlam ${ }^{1}$, J. M. MilleR ${ }^{2}$, E. M. CACKetT ${ }^{1}$ \\ Draft version July 23, 2018
}

\begin{abstract}
We systematically reanalyze two previous observations of the black hole $(\mathrm{BH}) \mathrm{GX} 339-4$ in the very high and intermediate state taken with XMM-Newton and Suzaku. We utilize up-to-date data reduction procedures and implement the recently developed, self-consistent model for X-ray reflection and relativistic ray tracing, RELXILL. In the very high and intermediate state, the rate of accretion is high and thus the disk remains close to the innermost stable circular orbit (ISCO). We require a common spin parameter and inclination when fitting the two observations since these parameters should remain constant across all states. This allows for the most accurate determination of the spin parameter of this galactic black hole binary from fitting the Fe $\mathrm{K} \alpha$ emission line and provides a chance to test previous estimates. We find GX 339-4 to be consistent with a near maximally spinning black hole with a spin parameter $a_{*}>0.97$ with an inclination of $36 \pm 4$ degrees. This spin value is consistent with previous high estimates for this object. Further, if the inner disk is aligned with the binary inclination, this modest inclination returns a high black hole mass, but they need not be aligned. Additionally, we explore how the spin is correlated with the power of the jet emitted but find no correlation between the two.
\end{abstract}

\section{INTRODUCTION}

GX 339-4 is an important source for accretion physics. It is a recurrent low mass X-ray binary system (LMXB) that has a prominent disk reflection spectrum. This galactic black hole binary (GBHB) has undergone four outbursts over the last twelve years, thus making it the one of the most active transient systems (Plant et al. 2014). Throughout its outbursts, GX 339-4 has been observed in the very high/soft state (VHS), intermediate state, and the low/hard state (LHS) followed by a very faint state, but has not yet been observed in the quiescent state (Hynes et al. 2003). See McClintock \& Remillard (2006) for a review on states. The mass of the $\mathrm{BH}$ and inclination are determined through interactions with the companion star. Through the analysis of emission lines during an outburst, Hynes et al. (2003) determined a mass function of $5.8 \pm 0.5 \mathrm{M}_{\odot}$, where the mass function can be expressed in the form of

$$
f(M)=\frac{M_{x} \sin i^{3}}{(1+q)^{2}}
$$

$M_{x}$ being the mass of the compact object, $q$ the mass ratio of the companion to the compact object, and $i$ the inclination. The inclination of the $\mathrm{BH}$ is still under some speculation since it may deviate from the inclination of the system itself, which is discussed in further detail in $\S 3$. The distance was determined using the optical/IR absorption along the line of sight to GX 339-4. It was found to lie between $6-15 \mathrm{kpc}$ (Hynes et al. 2004) though this value is generally quoted as a distance of $8 \mathrm{kpc}$ (Zdziarski et al. 2004).

GX 339-4 is an ideal source for applying new models that use the method of fitting $\mathrm{Fe} \mathrm{K} \alpha$ emission to estimate spin because of its prominent reflection features. The advantage of using the iron emission line is that it eliminates the need to know the mass or distance to the object. The determination of mass and distance is complicated by large uncertainties for most objects.

\footnotetext{
${ }^{1}$ Department of Physics \& Astronomy, Wayne State University, 666 W. Hancock St., Detroit, MI 48201, USA

${ }^{2}$ Department of Astronomy, University of Michigan, 1085 South University Ave, Ann Arbor, MI 48109-1107, USA
}

Models that allowed for the spin of a $\mathrm{BH}$ to be a variable parameter that could be constrained directly through the fitting of spectra emitted from an object were not available until 2004. However, line models did give important early indications of very high spin parameters (Miller et al. 2002). Since then, there have been numerous additive and multiplicative models developed to describe the different features that appear in the high energy X-ray band. These include models such as RELLINE (Dauser et al. 2010, 2013) and XILLVER (García \& Kallman 2010, 2013) among others. The latest of these models to be developed is the combination of the relativistic ray tracing kernel, RELLINE, and X-ray reflection code, XILLVER, into one self consistent model RELXILL (García et al. 2014).

GX 339-4 was estimated to harbor a near maximally spinning BH during an outburst in 2002-2003 (Miller et al. 2004a). Observations were taken by Chandra, XMM-Newton, and the Rossi X-Ray Timing Explorer (RXTE). This observation was among the first to use burst mode aboard $X M M-N e w t o n$, and some aspects of the reduction and systematics were unknown. Kirsch et al. (2006) later extensively went through analyzing burst mode data taken of the Crab Nebula in order to allow for a more conclusive extraction of spectra. In any case, Miller et al. (2004a) ruled out the possibility of this object being a non-spinning $\mathrm{BH}$ at more than the $8 \sigma$ confidence level from the iron $\left(\mathrm{Fe} \mathrm{K}_{\alpha}\right)$ emission line profile. They were able to estimate that the accretion disk extended in to $(2-3) R_{g}$, which translates to $a_{*} \geq 0.8-0.9$. Reis et al. (2008) reanalyzed the observations of GX 339-4 in the VHS and LHS (Miller et al. 2004a, 2006) which were observed by XMM-Newton. They used updated reflection models (REFLIONX and REFHIDEN) that were able to take into account blackbody emission and Compton scattering in the accretion disk that were not available prior. The results of their extensive modeling was a spin of $0.935 \pm 0.001$ which supported the previous findings.

These results were strengthened further by observations taken by Suzaku after an outburst that occurred during 20062007. Miller et al. (2008) fit the spectra obtained during the outburst with two other spectra taken by XMM while GX 339 4 was in a higher and lower state. The joint fit across states 
returned a BH with a spin parameter of $a_{*}=0.93 \pm 0.01$. This was again among the first observations in a burst mode, and the joint fitting procedures served as an important check. Later analysis of the Suzaku data by Yamada et al. (2009) found that the spectra may be piled up; however, a comprehensive study of pile-up by Miller et al. (2010) shows that pile-up only acts to narrow reflection features, and cause spin to be under-estimated.

Spin may be hard to determine in the low/hard state as can be seen in Dauser et al. (2014) and Fabian et al. (2014), therefore making it necessary to focus on the very high and intermediate states using new models to deliver the best possible spin measurement for GX 339-4. This focus on the VHS and intermediate state has to do with the location of the inner disk in relation to the ISCO. This is discussed further in $\S 3$. We reanalyze the observations from Miller et al. 2004a and Miller et al. 2008 using the latest data reduction procedures and model. This paper is formatted as follows: $\S 2$ describes the observations and data reduction process. $\S 3$ is the spectral analysis and results. $\S 4$ presents a discussion of the findings of this work.

\section{OBSERVATIONS AND DATA REDUCTION}

\subsection{XMM-Newton}

We reanalyze the $75.6 \mathrm{ks}$ observation of GX 339-4 in the very high state during revolution 0514 that began on 2002 September 29 at 09:06:42 UT. The EPIC-pn camera (Strüder et al. 2001) was operated in burst mode with a thin optical filter. The data were reduced using XMM-Newton Scientific Analysis System (SAS ver. 13.5.0). We also follow the guidelines for data reduction in burst mode from Kirsch et al. (2006). The data were reduced two different ways using the task EPFAST and again without the task since the use of EPFAST may alter the energy scale of the Fe $\mathrm{K}$ band (Walton et al. 2012). RAW space was set to RAWY $\leq 140$ and RAWX was 10 columns across (30-40). Above RAWY of 140 the determination of normalization becomes increasingly more affected based upon examination of figure two in Kirsch et. al. (2006). Although photon indices don't become affected until above RAWY $>160$, choosing to lower the limit ensures that $\Gamma$ is unaffected by pile up and a better determination of the normalization. In addition, the events were filtered to allow single and double events (PATTERN $\leq 4)$ while excluding bad pixels (FLAG==0).

Since the object is so bright, there was not a region available to extract a background. Most observations of GX 339. 4 with XMM-Newton have operated the EPIC-pn camera in "timing" mode, even when the source is not bright, serving to complicate background estimates. An entire Epic-pn CCD is exposed in timing mode. To estimate an upper limit on the background, we extracted an area equivalent to a Epicpn CCD (4.4 by 13.6 arcmin) from the MOS- 1 exposure of GX 339-4 obtained in obsID 0112900201 . We chose a region next to GX 339-4, and with a space density of background sources that is higher than average, as a kind of worstcase scenario. Extracting the data from this region, including point sources and any diffuse emission, gives a flux of $f=1.1 \times 10^{-12}$ ergs $\mathrm{cm}^{-2} \mathrm{~s}^{-1}$. In fact, this limit is much too high, as the extraction region that we used within timing mode is smaller than the full CCD. Even so, this flux is four orders of magnitude below the flux measured $\left(f=1.1576 \times 10^{-8} \mathrm{ergs} \mathrm{cm}^{-2} \mathrm{~s}^{-1}\right)$ from GX 339-4 in the observation of interest.
Response files were generated using SAS RMFGEN and ARFGEN commands. There are at least 20 counts per energy bin to allow the use of $\chi^{2}$ minimization when performing spectral fits. GX 339-4 is determined to be in the very high state at the time of this observation due to the spectral parameters found in Miller et al. (2004a) and the timing properties found in Homan et al. (2005a).

\subsection{RXTE}

RXTE simultaneously observed GX 339-4 on 2002 September 29 at 09:12:11 UT for $9.6 \mathrm{ks}$. There was not a simultaneous observation taken at the time of the Suzaku observation, but rather, there were four other observations taken on the same day for $3.5,3.41,3.4$, and $1.8 \mathrm{ks}$. We chose to use the standard products that are generated from standard modes after each observation as a guide for the photon index of the XMM-Newton and Suzaku observations. We added $0.6 \%$ systematic error to the spectra using the HEASOFT (ver. 6.15) sub package FTOOL GRPPHA task. The Proportional Counter Array (PCA; Jahoda et al. 2006) spectrum is generated from the standard 2 mode which covers 129 energy channels and has a 16s time resolution. The two High Energy X-ray Timing Experiment (HEXTE; Rothschild et al. 1998) spectra (from Cluster 0 and Cluster 1 ) have the same time resolution as the PCA and cover 129 spectral channels. Refer to The RXTE Cook Book for all these details. When performing spectral fits, the PCA was fit in the $3.0-25.0 \mathrm{keV}$ energy range and HEXTE fit the 20.0-100.0 keV energy range.

\subsection{Suzaku}

We reanalyze the observation of GX 339-4 in the intermediate state performed by Suzaku on 2007 February 12 beginning at 05:33:31 UT. This observation is classified as being in the intermediate state due to many properties that are consistent with previous results obtained in the intermediate states (Miller et al. 2004b). We processed the unfiltered event files through AEPIPELINE to produce clean event files for both the X-ray Imaging Spectrometer (XIS; Koyama et al. 2007) and Hard X-ray Telescope (HXD; Kokubun et al. 2007and Takahashi et al. 2007) instruments. XIS was operated in $0.3 \mathrm{~s}$ burst mode with the $1 / 4$ window option selected. HXD cameras were operated in the standard mode. All proper calibration products were determined and applied from the HEASARC Calibration Database (HXD20110913, XIS20120209, and XRT20110630).

We chose to use the two working 'front-illuminated' cameras, XIS0 and XIS3, in our analysis. The 'back-illuminated' instrument, XIS1, was not used due to calibration uncertainties as per Plant et al. (2015). New attitude files were created for both editing modes on each camera using AEATTCOR.SL 3 to account for the wobbling that Suzaku undergoes. The attitude files were then applied to the clean event files by using the FTOOL XISCOORD. Even though the 1/4 window and burst option were selected for this observation, there is still a significant amount of photon pileup in the data. Pileup causes the photon index of a spectrum to appear harder due to the inability of the detector to register individual events. Two or more events will be interpreted as a single event with a higher energy (see Miller et al. 2010 for a full description of the problem and consequences). Therefore, we use the RXTE observations that were taken on the same day as a

\footnotetext{
${ }^{3}$ http://space.mit.edu/ASC/software/suzaku/aeatt.html
} 
guide for the photon index of GX 339-4 in the $2.0-10.0 \mathrm{keV}$ energy range. We find that excluding data affected by pileup at the $8 \%$ level produces the proper spectral slope. We employ the tool PILEEST to determine the contours for this region for XIS0 and XIS3. We then exclude this region when using XIS XSELECT by using an annulus when extracting spectra. We used an outer radii of $7.5^{\prime}$ with an inner radius of $1.1^{\prime}$ and $1.4^{\prime}$ for XIS0 and XIS3 respectively. There were not available regions in the images to extract a background. Response files were created for each spectrum using the task XISRESP before combining the two XIS spectra together with the FTOOL ADDASCASPEC. Last, the combined spectrum was run through GRPPHA to require a minimum of 20 counts per energy bin like the XMM-Newton spectra.

The HXD/PIN was operated using the XIS nominal pointing. The proper response and non-X-ray background (NXB) files were obtained before executing the FTOOL HXDPINXBPI on the cleaned event. This tool creates a source spectrum that is corrected for dead time as well as a combined background (cosmic and non-X-ray) spectrum. A minimum of 20 counts per energy bin was required again. The GSO cleaned event file was run through the FTOOL HXDGSOXBPI after the appropriate NXB, spectral binning and additional ARF files were downloaded. The GSO NXB is already binned so the source spectrum must be binned accordingly. When performing spectral fits the XIS, PIN, and GSO were fit in the $1.0-10.0 \mathrm{keV}, 15.0-60.0 \mathrm{keV}$, and $70.0-600.0 \mathrm{keV}$ energy ranges respectively.

\section{SPECTRAL ANALYSIS AND RESULTS}

We use XSPEC version 12.8.1 (Arnaud 1996) in this work and errors are quoted at at least the $90 \%$ confidence level. We utilize the new RELXILL v0.1e modeling package (García et al.2014) to properly describe the reflection and relativistic effects within the spectra, specifically to fit the $\mathrm{Fe} \mathrm{K} \alpha$ emission line and determine the spin of this object. We combine RELXILL with the additive model DISKBB to account for the thermal component of the disk and the multiplicative model TBABS to account for absorption along the line of sight. The absorption column is fixed throughout this analysis to be $5.26 \times 10^{21} \mathrm{~cm}^{-2}$ in accordance with the findings by Dickey \& Lockman (1990). Note that GX 339-4 was in the "very high" state during the XMM-Newton observation and in the intermediate state for the Suzaku observation.

The inclination of GX 339-4 has not been determined conclusively. It is known that the orbital inclination has to be less than $60^{\circ}$ from the lack of eclipses present in the optical data (Cowley et al. 2002). Futher, it can not have a inclination of much less than $\sim 40^{\circ}$ in order to have a dynamical mass consistent with the findings of Hynes et al. (2003). Any lower of an inclination would give a BH with a mass greater than 20 $\mathrm{M}_{\odot}$. Yamada et al. (2009) found that the whole $25^{\circ}-45^{\circ}$ range is allowed at the $90 \%$ confidence level. On the other hand, the orbital inclination may not even be aligned with the inclination of the BH itself (see Maccarone 2002). We choose to allow the inclination to be free when fitting the spectra though we find it to be consistent with the $90 \%$ confidence range determined by Yamada et al. (2009).

We fit the two observations jointly in order to obtain better constraints on the parameters of greatest interest. The individual spectra provide fits of comparable quality, but do not give equally strong constraints on their own. Tying the Suzaku data with the EPIC-pn data provides a better estimate of the physics occurring within the source. The EPIC-

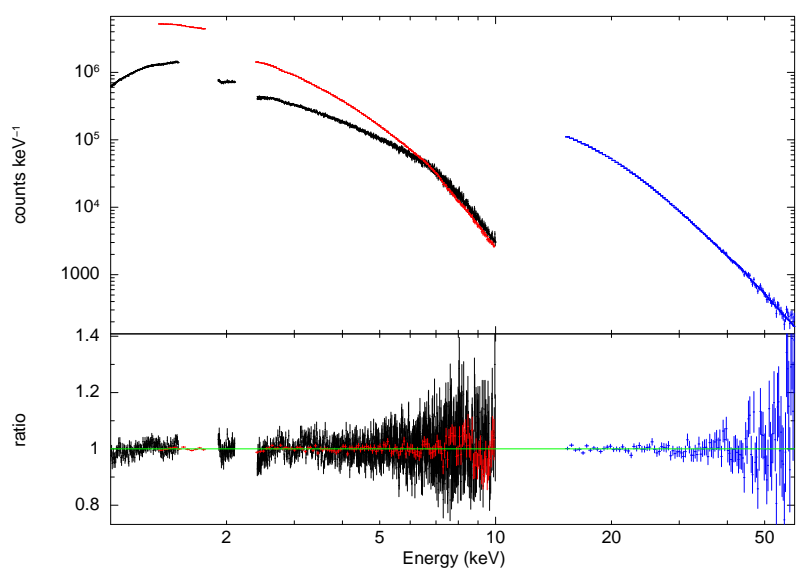

Figure 1. Simultaneous fit of Suzaku XIS (black) and XMM-Newton (red) with the addition of the HXD/PIN spectra (blue) extending to the higher energies. XIS data has been rebinned for plotting purposes. See Table 4 for parameter values. See Figure 2 for unfolded model spectrum.

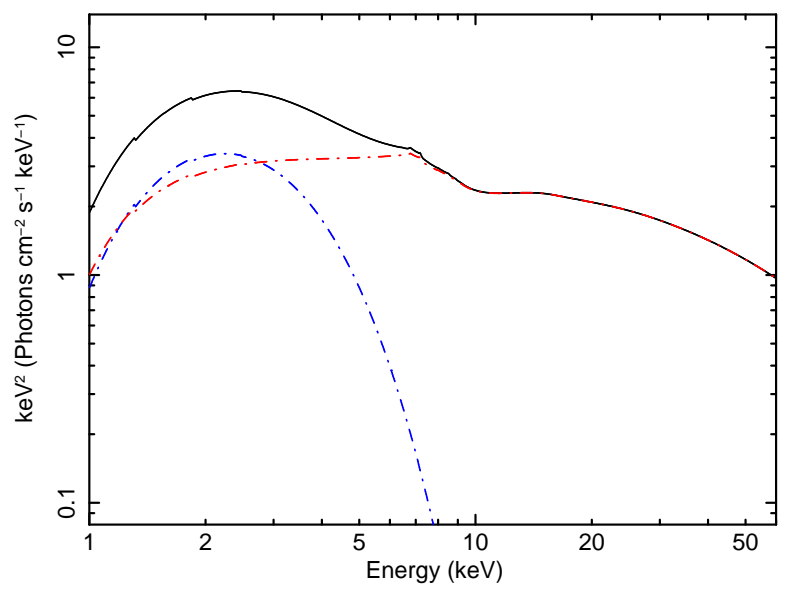

Figure 2. Unfolded model spectrum from Figure 1 for Suzaku XIS and HXD/PIN. The blue component corresponds to DISKBB. The red component illustrates RELXILL.

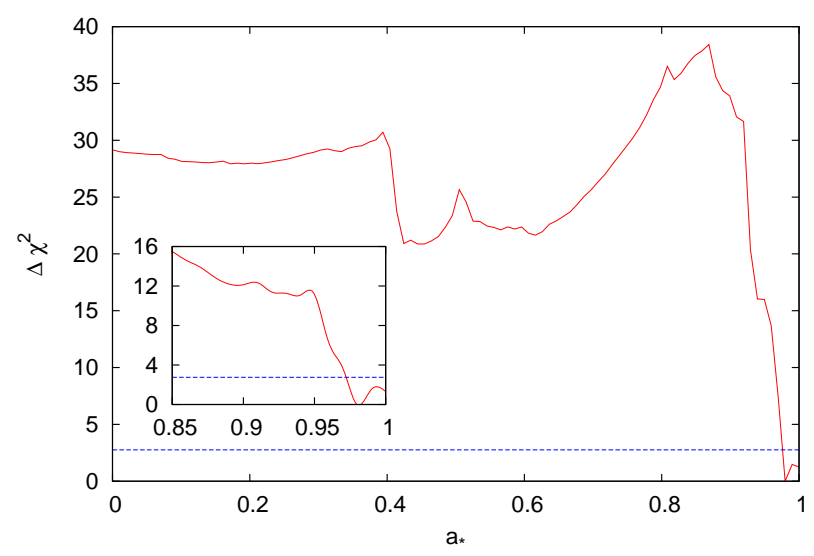

Figure 3. The change in goodness-of-fit versus spin taken over 100 evenly spaced steps generated with XSPEC "steppar". The spin was held constant at these steps while the other parameters were free to adjust. The inset shows a closer view of how $\chi^{2}$ changes for spin greater than 0.85 . The blue dotted line in both cases is the $90 \%$ confidence level. 
Table 1

Joint Fitting of Suzaku and XMM-Newton with HXD/PIN

\begin{tabular}{|c|c|c|c|}
\hline Component & Parameter & Suzaku & XMM-Newton \\
\hline TBABS & $N_{H}\left(10^{22}\right)^{\prime}$ & \multicolumn{2}{|c|}{0.526} \\
\hline DISKBB & $\begin{array}{l}T_{\text {in }}(\mathrm{keV}) \\
\text { norm }\end{array}$ & $\begin{array}{c}0.76 \pm 0.01 \\
2230_{-60}^{+110}\end{array}$ & $\begin{array}{c}0.80 \pm 0.01 \\
1640_{-80}^{+90}\end{array}$ \\
\hline \multirow[t]{16}{*}{ RELXILL } & $q_{\text {in }}$ & $9.9_{-6.9}^{+0.1}$ & $7 \pm 1$ \\
\hline & $q_{\text {out }}$ & $0.0002_{-0.0001}^{+0.9221}$ & $2.4_{-0.5}^{+0.7}$ \\
\hline & $R_{\text {break }}\left(R_{g}\right)$ & $5.3_{-2.6}^{+0.4}$ & $4.4 \pm 1.5$ \\
\hline & $a_{*}$ & \multicolumn{2}{|c|}{$>0.97$} \\
\hline & $i\left(^{\circ}\right)$ & \multicolumn{2}{|c|}{$36 \pm 4$} \\
\hline & $R_{i n}(I S C O)^{\prime}$ & \multicolumn{2}{|c|}{1} \\
\hline & $R_{\text {out }}\left(R_{g}\right)^{\prime}$ & \multirow{2}{*}{\multicolumn{2}{|c|}{$\begin{array}{c}400 \\
0\end{array}$}} \\
\hline & $z^{\prime}$ & & \\
\hline & $\Gamma$ & \multicolumn{2}{|r|}{$2.56 \pm 0.02$} \\
\hline & $\log (\xi)$ & $4.36 \pm 0.04$ & $4.7_{-0.3}^{+1.3}$ \\
\hline & $A_{F e}$ & \multicolumn{2}{|c|}{$4.8_{-0.7}^{+2.0}$} \\
\hline & $E_{\text {cut }}(k e V)$ & \multicolumn{2}{|c|}{$100_{-5}^{+0.7}$} \\
\hline & $f_{\text {refl }}$ & $2.4_{-1.7}^{+0.8}$ & $1.1_{-0.5}^{+1.7}$ \\
\hline & angleon' & \multicolumn{2}{|l|}{-1.8} \\
\hline & norm & $0.8 \pm 0.2$ & $0.9_{-0.4}^{+1.0}$ \\
\hline & $\chi_{\nu}^{2}$ (dof) & \multicolumn{2}{|c|}{$1.23(1472)$} \\
\hline
\end{tabular}

Note.-Errors are quoted at $\geq 90 \%$ confidence level. $N_{H}$ was fixed as the Dickey \& Lockman (1990) value. A constant was allowed to float between the XIS and HXD/PIN spectra. The XIS was frozen at the value of 1.0 and the HXD/PIN factor was fit at 0.857 . A Gaussian was modeled at $1.57 \mathrm{keV}$ with an equivalent width of $6.38 \times 10^{-5} \mathrm{keV}$ and $3.08 \times 10^{-2}$ normalization. The inner emissivity has been restricted between $3-10$. The outer emissivity has been restricted between $0-3$. The break radius was restricted between 3-6 $R_{g} . q_{i n}$ for the Suzaku spectra is not well constrained within restricted boundaries. Setting angleon $=1$ takes the inclination into account when modeling reflection. $\log (\xi)$ for XMM-Newton is consistent with the hard limit of 6 within the $90 \%$ confidence level.

pn spectrum has a limited bandwidth which may not render the proper spectral index. In order to obtain a better estimate of the photon index, $\Gamma$, we fit a grid of RELXILL models (for $a_{*}=0,0.5,0.99$ and $i<45$ ) with a blackbody disk component to the RXTE spectra for the simultaneous observation taken in 2002. A constant was allowed to float between the PCA and HEXTE. The photon index, $\Gamma$, was determined to be $2.56_{-0.03}^{+0.04}, 2.61 \pm 0.03$, and $2.62_{-0.05}^{+0.06}$ for the different values of fixed spin. To account for calibration uncertainties between the two missions, we restrict the photon index of the EPIC-pn spectrum to be within $\Delta \Gamma=0.1$ of the average value of $\Gamma=2.60$ when applying models. Again, the XMM-Newton data were reduced two different ways with and without the task EPFAST. We initially used the data that were reduced with EPFAST when modeling the data jointly with Suzaku. We then overlaid the spectrum that was generated without EPFAST onto the best fit to see if there was a significant difference between the two XMM-Newton spectra. The differences were very small so we chose to use the data generated with the task EPFAST. We ignore the 1.75-2.35 $\mathrm{keV}$ range in the XMM-Newton data rather than fitting multiple Gaussians to account for features of instrumental origin as per Plant et al. (2015). Additionally, we ignore below 1.3 $\mathrm{keV}$ due to residuals that were also identified by Reis et al. (2011), Hiemstra et al. (2011), and Plant et al. (2015). For the Suzaku data, we ignore the $1.5-1.9 \mathrm{keV}$ and $2.1-2.4 \mathrm{keV}$ energy ranges due to the poorly calibrated known $\mathrm{Si}-\mathrm{K}$ and $\mathrm{Au}-\mathrm{M}$ instrumental residuals.

As stated before, the model that we use to fit the spectra is TBABS*(DISKBB+RELXILL). In Table 1 are the values obtained when performing a joint fit between the XMM-Newton and Suzaku observation in the $1.0-10.0 \mathrm{keV}$ energy range and extended out into the higher energy range from $15.0-60.0 \mathrm{keV}$ with the spectra obtained from the HXD/PIN. The spin and inclination have been tied between the two observations because those should remain consistent between observations and state changes. The angleon parameter can be set to either 0 or 1 . Setting the angleon parameter to 0 causes the model to angle-average the X-ray reflection. Using an angleaveraged X-ray reflection model can bias the inclination to lower values (García et al. 2014). We make sure to set the angleon $=1$ so that the inclination is properly taken into account and not angle averaged. Figure 1 shows the simultaneous modeling of the spectra from XMM-Newton and Suzaku. Figure 2 shows the unfolded model spectrum for the Suzaku XIS and HXD/PIN as reference for what the models are accounting for the data.

We also assume in this model that the inner accretion disk is fully extended to the ISCO. Disk truncation is still a topic of debate, though there has been some recent evidence presented for truncation in the intermediate state (Tamura et al. 2012, Plant et al. 2015), it seems to be unlikely even in the low/hard states (see Miller et al. 2006; Rykoff et al. 2007; Reis et al. 2009, 2010). A strong indicator that the inner disk is extended down to the ISCO is the presence of high frequency quasiperiodic oscillations (Nowak 2000, Nespoli et al. 2003) in the intermediate state and the VHS. The frequencies are proportional with the orbits close to the ISCO (Strohmayer 2001, Miller et al. 2001, Homan et al. 2003, 2005b). We allow the emissivity, $q$, to be broken since constant emissivity throughout the disk is often thought to not be physically reasonable due to the difference in conditions in the accreting material closer to the BH (Wilkins \& Fabian 2012). For this reason we then allowed the inner emissivity $\left(q_{i n}\right)$ to vary between $3-10$ $R_{g}$ and the outer emissivity index $\left(q_{\text {out }}\right)$ to vary between $0-3$ $R_{g}$. The break radius was restricted to lie between $3-6 R_{g}$.

It can be seen that the model returns an acceptable fit to the data $\left(\chi^{2} / \operatorname{dof}=1816.36 / 1472\right)$. The high ionization parameter is consistent with those obtained in Reis et al. (2008) when looking at observations taken in the VHS and LHS. It was found that ionization parameter for the VHS was $\log (\xi)>4$ and in the LHS to be $\log (\xi) \approx 3$. It makes sense that the ionization parameter in the intermediate state would fall between the two yet be closer in value to the VHS since it was taken after the outburst had peaked. The ionization parameter value for the XMM-Newton spectrum is consistent with the hard limit of 6 within the $90 \%$ confidence level. The iron abundance was allowed to be free and returns a value that is $\approx 4$ times the solar abundance. This may be an overestimate that can be attributed to the RELXILL model. See García et al. 2014 and Kara et al. 2015 for further explanation. The inner emissivity for the Suzaku spectra is not well constrained but it is clearly not driving the spin result. The temperature of the disk is consistent with those found in Miller et al. (2008). The high reflection fraction suggest that the spectra is reflection dominated, but this is indicative of a BH with a high spin value (Dauser et al. 2014, Parker et al. 2014). The constant between the HXD/PIN and XIS is low with respect to the standard $\sim 1.16$, but this could be from extracting an annular region and having used an uncommon XIS mode.

\footnotetext{
4 JX-ISAS-SUZAKU-MEMO-2008-06 by Maeda et al.
} 
We find the inclination of GX 339-4 to be $36 \pm 4$ degrees. Using the mass function of $5.8 \pm 0.5 \mathrm{M}_{\odot}$ (Hynes et al. 2003) and a mass fraction of 0.125 (Muñoz-Darias et al. 2008) for this binary system with the inclination above suggests a large $\mathrm{BH}$ mass of $\sim 37.5 \mathrm{M}_{\odot}$. This applies if and only if the inclination of the binary system and the $\mathrm{BH}$ are aligned, but this need not be the case. A lower mass estimate can be achieved if the the inclination of the $\mathrm{BH}$ and the binary are misaligned. The true inclination is hard to determine as discussed earlier in this section. A higher inclination would also return a lower BH mass.

We obtain a spin parameter of $a_{*}>0.97$. This confirms the high spin estimates that have been found for GX 339-4 in the previous years. The maximum value for spin allowed for a BH is 0.998 (Thorne 1974). When constraining the error on spin we find that the maximum allowed value is within the $90 \%$ confidence level. We therefore quote the difference between the maximum allowed value and value found for spin as the upper bound. Figure 3 shows the change in $\chi^{2}$ when stepping the spin parameter from 0 to 0.998 using the "steppar" command in XSPEC.

\section{DISCUSSION}

We have found the spin of GX 339-4 to be a near maximally spinning BH with a spin parameter of $a_{*}>0.97$. The high reflection fraction also indicates a rapidly spinning $\mathrm{BH}$ (Dauser et al. 2014, Parker et al. 2014). This is consistent previous high spin estimates made by Miller et al. (2008) and Reis et al. (2008). The analysis made in previous works and our analysis performed with RELXILL are different in several respects:

- REFHIDEN allows for a different atmosphere, heated by a strong blackbody in the mid plane.

- RELXILL has a higher spectral resolution, improved atomic data, and includes more lines than prior models.

- RELXILL can be set to take the inclination angle into account when modeling X-ray reflection, whereas REFLIONX and REFHIDEN are angle-averaged.

It is important to account for systematic errors. Systematics owing to any violation of the test particle ISCO by an actual fluid disk are likely important, but appear to be small in recent numerical simulations (Reynolds \& Fabian 2008). We have found that the combination of atmospheric structure, model resolution, and inclination angle - each potentially an important source of systematic error - only produce $\sim 5 \%$ offsets in combination. In other words, they are not large sources of systematic error. It should be noted that inclination values that would keep the mass of the black hole in GX 339-4 below $10 M_{\odot}$ would necessitate eclipses, which are clearly not seen.

Given that GX339-4 is an important source, and that our results again point to a very high $\mathrm{BH}$ spin value, it is worth considering the role of spin on GX 339-4 and other stellarmass BHs. In particular, we look into where GX 339-4 falls according to existing relations between $\mathrm{BH}$ spin and jet power pertaining to $\mathrm{BH}$ binary (BHB) systems. One such relation in Narayan \& McClintock (2012) has found a near linear correlation for five BHBs with low mass companions and that produce ballistic jets during outburst. This appears to confirm the predictions of Blandford \& Znajek (1977) (BZ) where the relativistic jets are being powered by extracting energy from the
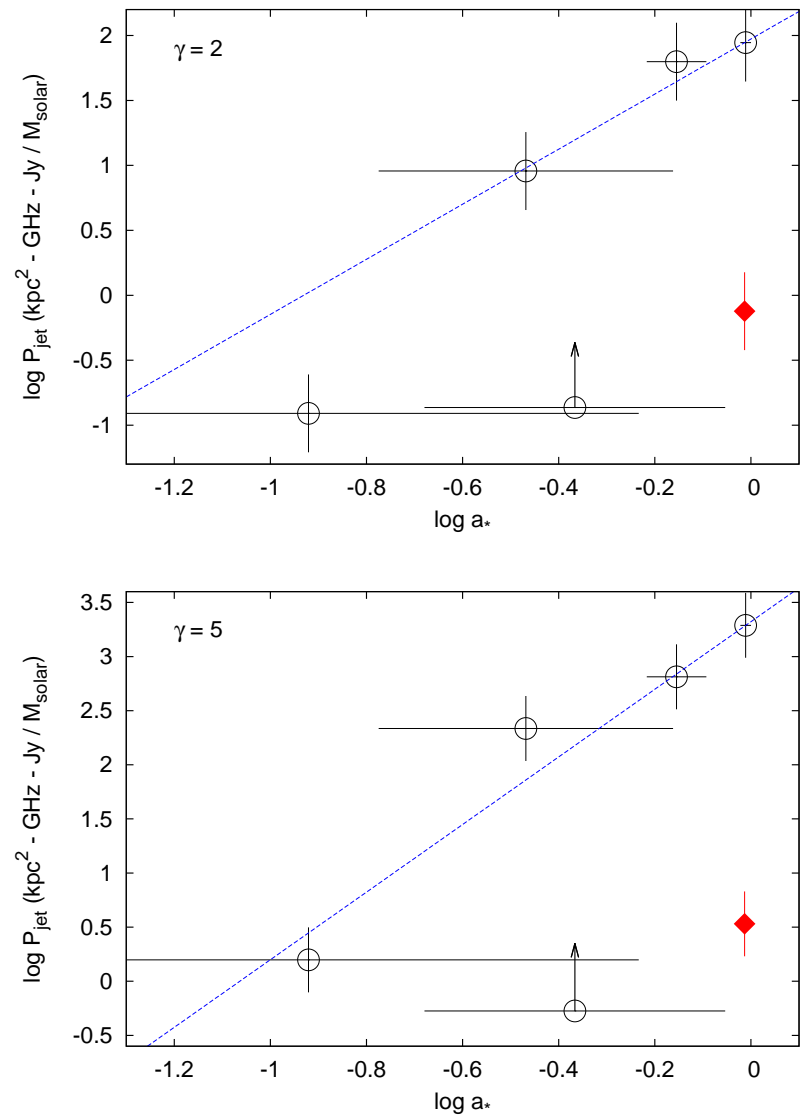

Figure 4. Black hole spin versus jet power. See Table 2 for values. GX 339-4 is given in red. Errors are plotted for the spin of GX 339-4 though they are to small to see. The top figure is for $\gamma=2$. The slope of the line is $2.12 \pm 0.75$ which is close to that predicted for the BZ effect. The lower figure is for $\gamma=5$ and has a slope of $3.12 \pm 0.39$.

angular momentum of the BH, though it was Penrose (1969) who first theorized this concept. Narayan \& McClintock do not include GX 339-4 in their correlation, but provide a rough estimate of the spin using their correlation. They estimate GX $339-4$ to have a spin between $0.2-0.5$ when assuming that the maximum observed radio flux is $55 \mathrm{mJy}$ (Gallo et al. 2004), which differs from our findings significantly. We take the observed flux density (at $5 \mathrm{GHz}$ ) and spin values that are presented in Narayan \& McClintock (2012) for four of the five sources: A0620-00, XTE J1550-564, GRO J1655-40, GRS $1915+105$. These spins were determined via the continuum fitting method. For the maximum observed radio flux density of GX 339-4 we use 55 mJy (Gallo et al. 2004). Recently, Morningstar \& Miller (2014) conducted a spin estimate for $4 \mathrm{U} 1543-47$ using the iron line emission similar to what we have done here for GX 339-4. We choose to use this recent estimate when looking into the relation between spin and jet power. The radio flux of 4U 1543-47 is seen as a lower limit for the jet power and is indicated as such in Figure 4 since it lacks sufficient radio data throughout the entirety of its outbursts (Park et al. 2004).

To change the observed fluxes into jet power we have to correct the fluxes for Doppler boosting. We use an equation from Mirabel \& Rodríguez (1999) to correct for this effect.

$$
S_{\text {cor }}=\frac{S_{o b s}}{\delta^{3-\alpha}}
$$


Table 2

Parameters for BH spin and Jet Power

\begin{tabular}{lcccc}
\hline \multicolumn{1}{c}{ BH Binary } & $a_{*}$ & $P_{\text {jet }}(\gamma=2)$ & $P_{\text {jet }}(\gamma=5)$ & ref \\
\hline A0620-00 & $0.12 \pm 0.19$ & 0.12 & 1.6 & 1 \\
XTE J1550-564 & $0.34 \pm 0.24$ & 9.1 & 220 & 1 \\
GRO J1655-40 & $0.7 \pm 0.1$ & 63 & 650 & 1 \\
GRS 1915+105 & $0.975 \pm 0.025$ & 88 & 1900 & 1 \\
4U 1543-47 & $0.43 \pm 0.31$ & 0.14 & 0.53 & 2 \\
GX 339-4 & $>0.97$ & 0.75 & 3.4 & 3 \\
\hline
\end{tabular}

Note.- The units of $P_{\text {jet }}$ are given in $\mathrm{kpc}^{2} \mathrm{GHz} \mathrm{Jy} \mathrm{M}_{\odot}^{-1}$. The reference refers to where the spin and/or observed maximum radio flux was taken. (1) Naravan \& McClintock 2012 (2) Morningstar \& Miller 2014 (3) Gallo et al. 2004.

where the Doppler factor $\delta=(\gamma(1-\beta \cos \theta))^{-1}, \gamma=$ $\left(\sqrt{1-\beta^{2}}\right)^{-1}$ is the Lorentz factor, $\beta=v / c$, and $\alpha$ is the radio spectral index. We use updated values for $\alpha$ from King et al. (2013a). These calculations were performed for two different values of Lorentz factors $(\gamma=2 \& \gamma=$ 5). $\gamma=2$ is chosen analogous with the work done in Narayan \& McClintock (2012), but since the Lorentz factor is difficult to determine we choose a higher value of $\gamma=5$ for comparison. We can then use the corrected flux for each object to estimate to the power of the jet being emitted from the $\mathrm{BH}$ by using the relation given Narayan \& McClintock (2012).

$$
P_{j e t}=D^{2} \nu S_{\nu} / M_{B H}
$$

Where $D$ is the distance to the object, $\nu$ is the observation frequency (taken to be $5 \mathrm{GHz}$ ), $S_{\nu}$ is the corrected radio flux density, and $M_{B H}$ is the mass of the BH. For GX 3394 we use $D=8 \mathrm{kpc}$ (Zdziarski et al. 2004) with $M_{B H}=$ $5.8 \pm 0.5 M_{\odot}$ (Hynes et al. 2003). The values for jet power can be seen in Table 2. Figure 4 shows the jet power in relation to the spin of the BHB on a logarithmic scale. The slope of weighted linear regression line for $\gamma=2$ is $2.12 \pm 0.75$, which is roughly consistent with that predicted of the BZ effect. However, the Lorentz factor is very difficult to constrain in many systems and is therefore largely uncertain (Fender 2003). When we allow the Lorentz factor to increase to 5, the slope of the best fit line becomes $3.12 \pm 0.39$. Yet, this steeper slope is still within $3 \sigma$ from the value at $\gamma=2$. This is not highly significant and can still be considered consistent with the BZ effect.

It can be seen in either case that GX 339-4 lies significantly below the predicted jet power via spin energy extraction for a near maximally spinning $\mathrm{BH}$. If we use the higher mass estimate of $\sim 37.5 \mathrm{M}_{\odot}$, when assuming the the inclination of the binary and $\mathrm{BH}$ are aligned, then the source is positioned even further away the from the correlation between $a_{*}$ and $P_{j e t}$. One explanation for the poor fit could be that the jet power is not correlated with the spin alone. Fender et al. (2010) and Russell et al. (2013) have found that spin and jet power are not correlated when a larger sample is used. King et al. (2013a,b) have investigated the role of spin in driving jets across the $\mathrm{BH}$ mass scale. They find that spin may set the maximum power in the jet, but that the mass accretion rate may act as a 'throttle' in setting the instantaneous jet power, as measured via radio flux. Both Russell et al. (2013) and King et al. (2013a) have found that a positive correlation between $P_{j e t}$ and $a_{*}$ is, at best, marginally significant for BHXBs $(\leq 2.3 \sigma$ confidence level). Our results are consistent with these findings. It is clear that the instantaneous jet power in GX 339-4 is not set by spin.

We would like to thank the anonymous referee for the helpful and constructive report. This research has made use of data and/or software provided by the High Energy Astrophysics Science Archive Research Center (HEASARC), which is a service of the Astrophysics Science Division at NASA/GSFC and the High Energy Astrophysics Division of the Smithsonian Astrophysical Observatory.

\section{REFERENCES}

Arnaud, K. A. 1996, in Astronomical Society of the Pacific Conference Series, Vol. 101, Astronomical Data Analysis Software and Systems V, ed. G. H. Jacoby \& J. Barnes, 17

Blandford, R. D., \& Znajek, R. L. 1977, MNRAS, 179, 433

Cowley, A. P., Schmidtke, P. C., Hutchings, J. B., \& Crampton, D. 2002, AJ, 123,1741

Dauser, T., García, J., Parker, M. L., Fabian, A. C., \& Wilms, J. 2014, MNRAS, 444, L100

Dauser, T., Garcia, J., Wilms, J., Böck, M., Brenneman, L. W., Falanga, M., Fukumura, K., \& Reynolds, C. S. 2013, MNRAS, 430, 1694

Dauser, T., Wilms, J., Reynolds, C. S., \& Brenneman, L. W. 2010, MNRAS, 409, 1534

Dickey, J. M., \& Lockman, F. J. 1990, ARA\&A, 28, 215

Fabian, A. C., Parker, M. L., Wilkins, D. R., Miller, J. M., Kara, E., Reynolds, C. S., \& Dauser, T. 2014, MNRAS, 439, 2307

Fender, R. P. 2003, MNRAS, 340, 1353

Fender, R. P., Gallo, E., \& Russell, D. 2010, MNRAS, 406, 1425

Gallo, E., Corbel, S., Fender, R. P., Maccarone, T. J., \& Tzioumis, A. K. 2004, MNRAS, 347, L52

García, J. et al. 2014, ApJ, 782, 76

García, J., Dauser, T., Reynolds, C. S., Kallman, T. R., McClintock, J. E. Wilms, J., \& Eikmann, W. 2013, ApJ, 768, 146

García, J., \& Kallman, T. R. 2010, ApJ, 718, 695

Hiemstra, B., Méndez, M., Done, C., Díaz Trigo, M., Altamirano, D., \& Casella, P. 2011, MNRAS, 411, 137

Homan, J., Buxton, M., Markoff, S., Bailyn, C. D., Nespoli, E., \& Belloni, T. 2005a, ApJ, 624, 295

Homan, J., Klein-Wolt, M., Rossi, S., Miller, J. M., Wijnands, R., Belloni, T., van der Klis, M., \& Lewin, W. H. G. 2003, ApJ, 586, 1262

Homan, J., Miller, J. M., Wijnands, R., van der Klis, M., Belloni, T., Steeghs, D., \& Lewin, W. H. G. 2005b, ApJ, 623, 383

Hynes, R. I., Steeghs, D., Casares, J., Charles, P. A., \& O’Brien, K. 2003, ApJ, 583, L95

-. 2004, ApJ, 609, 317

Jahoda, K., Markwardt, C. B., Radeva, Y., Rots, A. H., Stark, M. J., Swank,

J. H., Strohmayer, T. E., \& Zhang, W. 2006, ApJS, 163, 401

Kara, E. et al. 2015, ArXiv e-prints

King, A. L., Miller, J. M., Gültekin, K., Walton, D. J., Fabian, A. C.,

Reynolds, C. S., \& Nandra, K. 2013a, ApJ, 771, 84

King, A. L. et al. 2013b, ApJ, 762, 103

Kirsch, M. G. F. et al. 2006, A\&A, 453, 173

Kokubun, M., et al. 2007, PASJ, 59, 53

Koyama, K., et al. 2007, PASJ, 59, 23

Maccarone, T. J. 2002, MNRAS, 336, 1371

McClintock, J. E., \& Remillard, R. A. 2006, Black hole binaries, ed.

W. H. G. Lewin \& M. van der Klis, 157-213

Miller, J. M. et al. 2010, ApJ, 724, 1441

-. 2004a, ApJ, 606, L131

-. 2002, ApJ, 570, L69

Miller, J. M., Homan, J., Steeghs, D., Rupen, M., Hunstead, R. W.,

Wijnands, R., Charles, P. A., \& Fabian, A. C. 2006, ApJ, 653, 525

Miller, J. M. et al. 2004b, ApJ, 601, 450

-. 2008, ApJ, 679, L113

- 2001, ApJ, 563, 928

Mirabel, I. F., \& Rodríguez, L. F. 1999, ARA\&A, 37, 409

Morningstar, W. R., \& Miller, J. M. 2014, ApJ, 793, L33

Muñoz-Darias, T., Casares, J., \& Martínez-Pais, I. G. 2008, MNRAS, 385, 2205

Narayan, R., \& McClintock, J. E. 2012, MNRAS, 419, L69 
Nespoli, E., Belloni, T., Homan, J., Miller, J. M., Lewin, W. H. G., Méndez, M., \& van der Klis, M. 2003, A\&A, 412, 235

Nowak, M. A. 2000, MNRAS, 318, 361

Park, S. Q. et al. 2004, ApJ, 610, 378

Parker, M. L. et al. 2014, MNRAS, 443, 1723

Penrose, R. 1969, Nuovo Cimento Rivista Serie, 1, 252

Plant, D. S., Fender, R. P., Ponti, G., Muñoz-Darias, T., \& Coriat, M. 2014, MNRAS, 442, 1767

-. 2015, A\&A, 573, A120

Reis, R. C., Fabian, A. C., \& Miller, J. M. 2010, MNRAS, 402, 836

Reis, R. C., Fabian, A. C., Ross, R. R., \& Miller, J. M. 2009, MNRAS, 395, 1257

Reis, R. C., Fabian, A. C., Ross, R. R., Miniutti, G., Miller, J. M., \& Reynolds, C. 2008, MNRAS, 387, 1489

Reis, R. C. et al. 2011, MNRAS, 410, 2497

Reynolds, C. S., \& Fabian, A. C. 2008, ApJ, 675, 1048
Rothschild, R. E. et al. 1998, ApJ, 496, 538

Russell, D. M., Gallo, E., \& Fender, R. P. 2013, MNRAS, 431, 405

Rykoff, E. S., Miller, J. M., Steeghs, D., \& Torres, M. A. P. 2007, ApJ, 666, 1129

Strohmayer, T. E. 2001, ApJ, 552, L49

Strüder, L., et al. 2001, A\&A, 365, L18

Takahashi, T., et al. 2007, PASJ, 59, 35

Tamura, M., Kubota, A., Yamada, S., Done, C., Kolehmainen, M., Ueda, Y., \& Torii, S. 2012, ApJ, 753, 65

Thorne, K. S. 1974, ApJ, 191, 507

Walton, D. J., Reis, R. C., Cackett, E. M., Fabian, A. C., \& Miller, J. M. 2012, MNRAS, 422, 2510

Wilkins, D. R., \& Fabian, A. C. 2012, MNRAS, 424, 1284

Yamada, S. et al. 2009, ApJ, 707, L109

Zdziarski, A. A., Gierliński, M., Mikołajewska, J., Wardziński, G., Smith, D. M., Harmon, B. A., \& Kitamoto, S. 2004, MNRAS, 351, 791 